\title{
Numerical method for calculating nonlinear sound propagation in full acoustic field
}

\author{
Kei Fujisawa ${ }^{1, *}$ and Akira Asada ${ }^{2}$ \\ ${ }^{1}$ Department of Ocean Technology, Policy, and Environment, The University of Tokyo, \\ 4-6-1 Komaba, Meguro-ku, Tokyo, 153-8505 Japan \\ ${ }^{2}$ Institute of Industrial Science, The University of Tokyo, \\ 4-6-1 Komaba, Meguro-ku, Tokyo, 153-8505 Japan
}

(Received 12 February 2015, Accepted for publication 12 March 2015)

Keywords: Numerical method, Nonlinear sound propagation, Navier-Stokes equation,

Underwater acoustics, Full acoustic field, KZK equation

PACS number: 43.25.Cb [doi:10.1250/ast.36.438]

\section{Introduction}

Nonlinear sound propagation in the far-field acoustics has been studied by numerically solving the KhokhlovZaboloskya-Kuznetsov (KZK) equation [1-3], which is derived from the fluid dynamic equation under the assumption of parabolic approximation [4]. It is well known that the approach based on the KZK equation is efficient enough in the far-field acoustics, whereas it is not accurate enough in the near sound source owing to the parabolic nature of the equation [5,6]. Therefore, the approach based on the KZK equation cannot be applied to nonlinear acoustics in the near field, where the reflection, refraction and interference of sound play an important role in the nonlinear sound propagation. Such a situation can be seen in applications, such as beam focusing by an acoustic phased array, and an acoustic lens.

On the other hand, full-field (near- and far-field) acoustics can be studied by the fluid dynamic equation based approach [7-9]. This approach uses the compressible form of the Navier-Stokes equation and is solved numerically by the finite difference time domain (FDTD) method, although it requires a large amount of computational time even for a small target area of computation. Therefore, it is difficult to simulate the full-field acoustics using this approach, especially in the huge target acoustic field of underwater acoustics, where full acoustic fields larger than $1 \mathrm{~m}$ in axial length must be solved to study the nonlinear sound propagation from parametric and phased arrays.

The purpose of this study is to devise a numerical method for nonlinear sound propagation in the full acoustic field with reasonable computational cost by combining two approaches of the Navier-Stokes and KZK equations.

\section{Combined numerical method for full acoustic field}

In this section, the numerical method for full-field acoustics is described, which combines the Navier-Stokes equation in the near-field and the KZK equation in the farfield.

\subsection{Fluid dynamic model}

One of the numerical approaches applicable to nonlinear sound propagation in full acoustic field is based on the

\footnotetext{
*e-mail: fujisawa_ocean_eng@yahoo.co.jp
}

Navier-Stokes equation. This approach considers the nonlinearity, dissipation, thermal conduction through the propagation in thermal viscous fluid by solving the continuity equation (1), the compressible form of the Navier-Stokes equation (2), the entropy balance equation (3), and the state equation (4).

$$
\begin{gathered}
\frac{D \rho}{D t}+\rho \nabla \cdot \boldsymbol{u}=0 \\
\rho \frac{D \boldsymbol{u}}{D t}+\nabla \cdot(P \boldsymbol{1}-\boldsymbol{\tau})=0 \\
\rho T \frac{D S}{D t}=\kappa \nabla^{2} T+\boldsymbol{\tau}: \nabla \boldsymbol{u} \\
P^{*}=c_{0}^{2} \rho^{*}+\frac{c_{0}^{2} B}{2 \rho_{0} A} \rho^{* 2}+\left(\frac{\partial P}{\partial s}\right)_{\rho, 0} S^{*}
\end{gathered}
$$

where $\tau$ : deviatoric stress tensor, $c_{0}$ : sound speed, $t$ : time, $B / A$ : parameter of nonlinearity, $P$ : pressure, $S$ : entropy, $T$ : temperature, $\kappa$ : thermal conductivity, $\mu$ : shear viscosity, $\xi$ : bulk viscosity, $\rho$ : density and $\boldsymbol{u}$ : velocity vector. The index $*$ expresses the fluctuation from the initial condition $\left(P^{*}=\right.$ $\left.P-P_{0}, \quad \rho^{*}=\rho-\rho_{0}, \quad S^{*}=S-S_{0}\right)$ and the subscript 0 indicates the reference fluid properties at $T=298 \mathrm{~K}$ in atmospheric pressure. These equations are solved by a finite-difference time domain (FDTD) based method using staggered grids. It should be mentioned that the 2 nd order difference scheme is applied to the acoustic terms in space and time, the 1st order upwind difference scheme is used for the advection term, and the 2nd-order central difference scheme is used for the dissipative terms. The validation of the present simulation code is confirmed in comparison with the reported result in the literature [8].

\subsection{KZK model}

The KZK equation is the parabolic equation that is commonly used in nonlinear sound propagation considering the nonlinearity, diffraction, and diffusivity of sound propagation.

$$
\frac{\partial^{2} p}{\partial t^{\prime} \partial z}-\frac{c_{0}}{2} \nabla_{\perp}^{2} p-\frac{\gamma}{2 c_{0}{ }^{3}} \frac{\partial^{3} p}{\partial t^{\prime 3}}-\frac{\beta}{2 \rho_{0} c_{0}{ }^{3}} \frac{\partial^{2} p^{2}}{\partial t^{\prime 2}}=0
$$

where $t^{\prime}=t-z / c_{0}, \beta$ : nonlinear coefficient, and $\gamma$ : diffusivity of sound. This equation expresses the nonlinear wave 


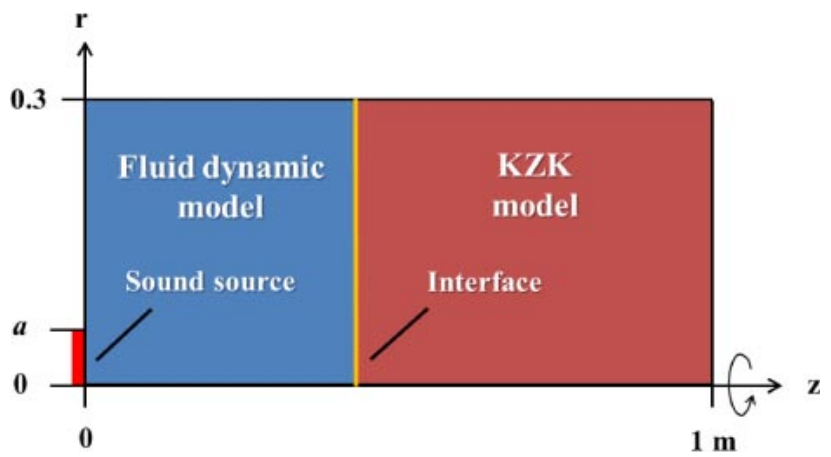

Fig. 1 Schematic description of computational area.

propagation in fluid by $\beta$ and $\gamma$. The second term of Eq. (5) represents the diffraction, the third term is the dissipation, and the fourth term is the nonlinearity in sound propagation. The equation can be solved numerically after converting it into Fourier series equations by the implicit finite-difference method [3].

\subsection{Interface boundary condition}

The fluid dynamic model in the near field is solved in the time domain, while the KZK model in the far field is solved in the frequency domain. To smoothly connect the sound pressure at the interface, the boundary condition of sound pressure is evaluated from the fluid dynamic model and is transferred to the KZK model at the interface. Note that the interface can be located near the sound source, where the parabolic assumption is acceptable.

\section{Numerical simulation of sound propagation from a sound source}

The combined model is applied to the full acoustic field of sound propagation in water emitted from a sound source $(z=0)$, as shown in Fig. 1. The computational area is $0.3 \mathrm{~m}$ in radius and $1 \mathrm{~m}$ in axial distance, and the radius of sound source is $\mathrm{a}=0.01 \mathrm{~m}$. The fluid properties of water used in the computation are as follows: sound velocity $c_{0}=1496 \mathrm{~m} / \mathrm{s}$, density $\rho_{0}=0.997 \times 10^{3} \mathrm{~kg} / \mathrm{m}^{3}$, shear viscosity $\mu=0.89 \times$ $10^{-3} \mathrm{~Pa} \mathrm{~s}$, bulk viscosity $\xi=2.4 \times 10^{-3} \mathrm{Pas}$, thermal conductivity $\kappa=0.61 \mathrm{~W} /(\mathrm{m} \mathrm{K})$, nonlinear parameter $B / A=5$, nonlinearity coefficient $\beta=3.5$, and diffusivity of sound $\gamma=3.6 \times 10^{-6} \mathrm{~m}^{2} / \mathrm{s}$. The sound pressure fluctuation at the sound source is given by the sinusoidal frequency of $1 \mathrm{MHz}$ and amplitude of $0.1 \mathrm{MPa}$. The outer boundary condition is prescribed by the absorbing boundary to minimize the reflection from the boundary for the fluid dynamic model [10] and KZK model [11]. It should be mentioned that the interface of the combined model is located at $z=0.052 \mathrm{~m}$ ( $=35 \lambda, \lambda$ : wavelength) from the sound source, where the parabolic approximation is acceptable. However, the selection of the interface boundary in the far field, $z>0.052 \mathrm{~m}$, does not change the sound pressure distribution.

\section{Results and discussion}

Figure 2 shows the computational results of the fundamental and second harmonics of the nonlinear sound propagation in the axial direction, which are obtained from

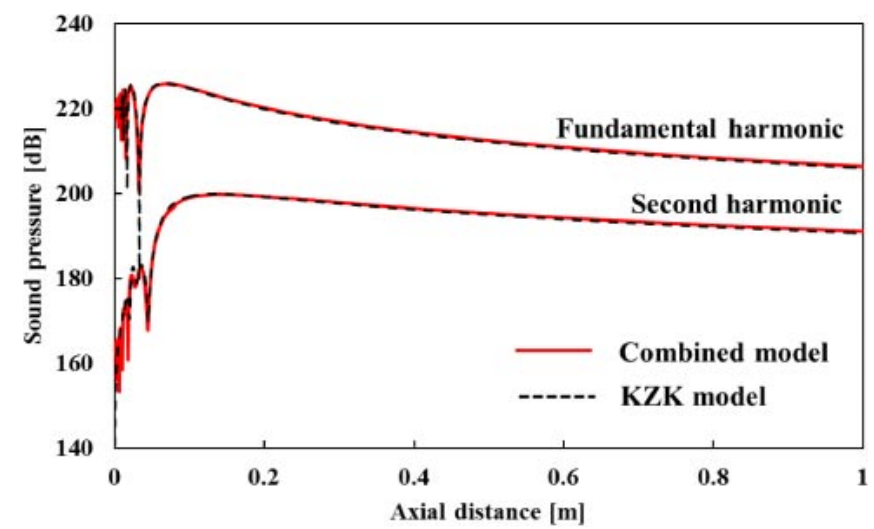

Fig. 2 Sound pressure distribution in full acoustic field on beam axis $(0-1 \mathrm{~m})$.

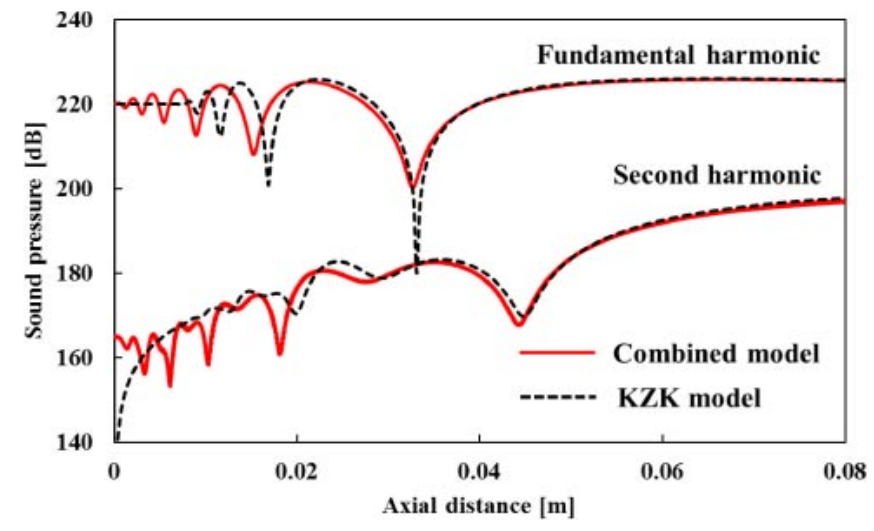

Fig. 3 Sound pressure distribution near sound source $(0-0.08 \mathrm{~m})$.

the numerical simulation by the combined model and that by the KZK model. The numerical result indicates that the sound pressure level of the combined model and that of the KZK model agree closely in the far field, while the computations in the near field deviate from each other owing to the parabolic approximation of the KZK model. Note that the sound pressure level of the combined model is smoothly connected at the interface, which suggests smooth data transfer of the sound pressure at the interface of the combined model.

The close-up view of the near field is shown in Fig. 3. It is clear that the KZK model shows a minor variation in sound pressure, whereas the combined model shows a clear variation in sound pressure near the sound source. Such variations were similarly observed in the near field of the sound source by Nomura et al. [7], who simulated the nonlinear sound pressure propagation using a fluid dynamic model and compared their findings with numerical result of the KZK model.

The present computation was carried out using the grids (fluid dynamic model: $3743 \times 16040$, KZK model: $14974 \times$ $1604)$ in the axial and radial directions, respectively, on a PC with Intel(R) Core(TM) i7-4770 CPU $3.40 \mathrm{GHz}$ with OpenMP. In this case, the computational time is 14 hours with the combined model, while the expected CPU time is 
expected to be more than 2,400 hours with the fluid dynamic model. These results indicate that the combined model shows a higher accuracy than the KZK model in the near field and has a much lower computational cost than the fluid dynamic model. Therefore, the combined model is an efficient tool for the study of nonlinear sound propagation in underwater acoustics.

\section{Conclusion}

A new numerical method is described for solving the nonlinear sound propagation in a full acoustic field with reasonable computational time. This method combines the fluid dynamic model in the near field and the KZK model in the far field. The validation of this numerical method and its efficiency are proved by applying a nonlinear sound propagation from a sound source.

\section{References}

[1] E. A. Zabolotskaya and R. V. Khokhlov, "Quasi-plane waves on the nonlinear acoustics of confined beams," Sov. Phys. Acoust., 15, 35-40 (1969).

[2] V. P. Kuznetsov, "Equation of nonlinear acoustics," Sov. Phys. Acoust., 16, 467-470 (1971).

[3] S. I. Aanonsen, "Numerical computation of the nearfield of a finite amplitude sound beam," Tech Rep. No. 73, Department of Mathematics, University of Bergen, Bergen, Norway
(1983).

[4] P. J. Westervelt, "Parametric acoustic array," J. Acoust. Soc. Am., 35, 535-537 (1963).

[5] V. F. Humphrey, "Nonlinear propagation in ultrasonic fields: measurements, modelling and harmonic imaging," Ultrasonics, 38, 267-272 (2000).

[6] T. Kamakura, M. Akiyama and K. Aoki, "A higher-order parabolic equation for describing nonlinear propagation of ultrasound beams," Acoust. Sci. \& Tech., 25, 163-165 (2004).

[7] H. Nomura, C. M. Hedberg and T. Kamakura, "Numerical simulation of parametric sound generation and its application to length-limited sound beam," Appl. Acoust., 73, 1231-1238 (2012).

[8] H. Nomura, H. Adachi, T. Kamakura and T. G. Clement, "Feasibility of low-frequency directive sound source with high range resolution using pulse compression technique," Jpn. J. Appl. Phys., 53, $07 \mathrm{KC} 03$ (2014).

[9] K. Fujisawa, "Numerical study on nonlinear sound propagation for parametric array," J. Flow Control Meas. Vis., 2, 120-124 (2014).

[10] G. Mur, "Absorbing boundary condition for the finite-difference approximation of the time-domain electromagnetic-field equation," IEEE Trans. Electromagn. Compat., 4, 377-382 (1981).

[11] F. Dagrau, M. Renier, R. Marchano and F. Coulouvrat, "Acoustic shock wave propagation in a heterogeous medium: A numerical simulation beyond the parabolic approximation," J. Acoust. Soc. Am., 130, 20-32 (2011). 